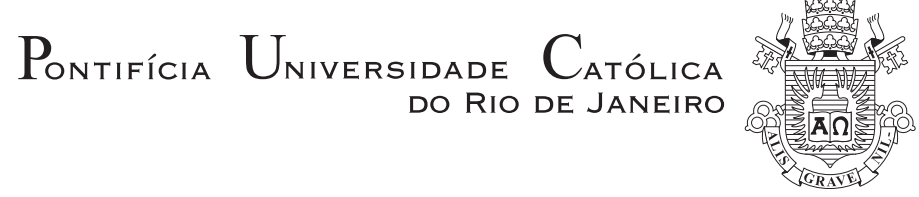

Roberta de Queiroz Lima

\title{
Análise de Vibrações Estocásticas em Sistemas Mecânicos
}

Dissertação de Mestrado

Dissertação apresentada ao Programa de Pós-graduação em Engenharia Mecânica da PUC-Rio como requisito parcial para obtenção do título de Mestre em Engenharia Mecânica.

Orientador: Prof. Rubens Sampaio Filho 


\title{
Roberta de Queiroz Lima
}

\section{Análise de Vibrações Estocásticas em Sistemas Mecânicos}

Dissertação apresentada como requisito parcial para obtenção do grau de Mestre pelo Programa de Pós-graduação em Engenharia Mecânica da PUC-Rio. Aprovada pela Comissão Examinadora abaixo assinada.

\author{
Prof. Rubens Sampaio Filho \\ Orientador \\ Departamento de Engenharia Mecânica - PUC-Rio \\ Prof. Márcio da Silveira Carvalho \\ Departamento de Engenharia Mecânica — PUC-Rio \\ Prof. Thiago Gamboa Ritto \\ Departamento de Engenharia Mecânica — UFRJ
}

Prof. Edson Luiz Cataldo Ferreira Departamento de Matemática Aplicada - UFF

Prof. Mario Raúl Escalante Departamento de Ciências Básicas - UTN-FRCON, UTN-FRCU

Prof. José Eugênio Leal Coordenador Setorial do Centro Técnico Científico - PUC-Rio 
Todos os direitos reservados. É proibida a reprodução total ou parcial do trabalho sem autorização da universidade, do autor e do orientador.

\section{Roberta de Queiroz Lima}

Graduou-se em Engenharia Mecânica pela Pontifícia Universidade Católica do Rio de Janeiro em 2009, tendo dedicado um ano e meio a projetos de iniciação científica. Publicou um trabalho junto com seu orientador durante o Mestrado no MECOM 2010 e, teve um artigo aceito para publicação no COBEM 2011. Atualmente, dedica-se ao curso de Doutorado em Engenharia Mecânica na PUC-Rio.

Ficha Catalográfica

Lima, Roberta de Queiroz

Análise de Vibrações Estocásticas em Sistemas Mecânicos / Roberta de Queiroz Lima; orientador: Rubens Sampaio Filho. - Rio de Janeiro : PUC-Rio, Departamento de Engenharia Mecânica, 2011.

v., $148 \mathrm{f:} \mathrm{il.} \mathrm{;} 29,7 \mathrm{~cm}$

1. Dissertação (mestrado) - Pontifícia Universidade Católica do Rio de Janeiro, Departamento de Engenharia Mecânica.

Inclui referências bibliográficas.

1. Engenharia Mecânica - Tese. 2. Geração de Amostras de Variáveis e Vetores Aleatórios; Método de Monte Carlo com Cadeia de Markov; Vibrações Estocásticas; Método de Galerkin. I. Filho, Rubens Sampaio. II. Pontifícia Universidade Católica do Rio de Janeiro. Departamento de Engenharia Mecânica. III. Título. 


\section{Agradecimentos}

À minha família pelo apoio, compreensão e carinho durante toda a minha vida.

Agradeço ao meu orientador, Rubens, pela amizade, estímulo e ensinamentos que contribuíram muito para a minha formação profissional e pessoal.

Agradeço à PUC-Rio e às agências CNPQ, CAPES e, FAPERJ pelo auxílio concedido no desenvolvimento desse trabalho. E finalmente agradeço a todos os amigos e professores do Departamento de Engenharia Mecânica da PUC-Rio, UFRJ e UFF que me sempre me incentivaram e apoiaram. 


\section{Resumo}

Lima, Roberta de Queiroz; Filho, Rubens Sampaio. Análise de Vibrações Estocásticas em Sistemas Mecânicos. Rio de Janeiro, 2011. 148p. Dissertação de Mestrado - Departamento de Engenharia Mecânica, Pontifícia Universidade Católica do Rio de Janeiro.

A parte inicial da dissertação apresenta uma discussão sobre o método de Monte Carlo e sobre geradores de realizações de variáveis aleatórias e vetores aleatórios. São analisados geradores baseados em: congruência linear, Método da Transformada Inversa e algoritmo de Metrópolis - Hastings (método de Monte Carlo com Cadeia de Markov MCMC). Posteriormente, são apresentadas técnicas para a análise de vibrações estocásticas em sistemas mecânicos com um grau de liberdade, múltiplos graus de liberdade e contínuos. As propriedades dos sistemas são consideradas determinísticas e, as forças são caracterizadas por processos estocásticos. Ao longo das seções do trabalho, é mostrado como características da resposta do sistema em deslocamento (média, autocorrelação e densidade espectral) podem ser obtidas a partir das informações sobre o forçamento. A Transformada de Fourier, a função densidade espectral e a função de transferência são importantes ferramentas utilizadas no estudo. No caso de sistemas com múltiplos graus de liberdade, a análise é feita por dois métodos: Resposta ao Impulso e Modos Normais. Nos sistemas contínuos, a análise é feita através do Método de Monte Carlo. Para isso, foi desenvolvido um exemplo de um sistema mecânico contínuo submetido a dois carregamentos caracterizados por processos estocásticos. Nesse exemplo, o MCMC foi utilizado para gerar amostras dos forçamentos. Em cada realização, o sistema foi discretizado através do Método dos Elementos Finitos e uma aproximação para solução do problema foi obtida utilizando o Método de Galerkin. Nessa parte da dissertação são apresentados a formulação fraca da equação de movimento da viga e o modelo reduzido. Ao longo de todo o trabalho são mostrados exemplos e resultados obtidos através de rotinas desenvolvidas em MATLAB.

\section{Palavras-chave}

Geração de Amostras de Variáveis e Vetores Aleatórios; Método de Monte Carlo com Cadeia de Markov; Vibrações Estocásticas; Método de Galerkin. 


\section{Abstract}

Lima, Roberta de Queiroz; Filho, Rubens Sampaio (Advisor). Random Vibration Analysis of Mechanical Systems. Rio de Janeiro, 2011. 148p. MSc. Dissertation — Departamento de Engenharia Mecânica, Pontifícia Universidade Católica do Rio de Janeiro.

The initial part of the work presents a discussion about the Monte Carlo Method and about the generation of samples of random variables and random vectors. Generators based on linear congruential, on the Inverse Transform Method and, on Metropolis - Hastings algorithm (Markov Chain Monte Carlo Method MCMC) are analyzed. Later, techniques for vibrations analysis of single-degree of freedom, multi-degree of freedom and continuous stochastic mechanical systems are presented. The system properties are considered deterministic and, the forces are characterized by random processes. Throughout the work sections, it is shown how the system's displacement response (mean, autocorrelation and spectral density) can be characterized from the force informations. The Fourier transform, the spectral density function and transfer function are powerful tools used in the study. In the case of multi-degree of freedom systems, the analysis is done by two methods: Impulse Response and Normal modes. For continuous systems, it is made by Monte Carlo method. An example of one continuous mechanical system with two random loads were developed. In this example, MCMC is used to generate samples of the forces. For each realization, the problem is discretized by the Finite Element Method and one approximation of the solution is obtained by the Galerkin Method. In this part of the work, the weak formulation of the motion equation of the vibrating beam and the reduced model are presented. Throughout the work many routines in MATLAB were developed.

\section{Keywords}

Generation of samples of random variables and random vectors; Markov Chain Monte Carlo Method; Random Vibration; Galerkin Method. 


\section{Sumário}

1 Introdução $\quad 13$

1.1 Objetivos do trabalho 13

$\begin{array}{lll}1.2 & \text { Organização do trabalho } & 14\end{array}$

2 Método de Monte Carlo $\quad \mathbf{1 6}$

$\begin{array}{lll}2.1 \text { Introdução } & 16\end{array}$

2.2 Gerador de Amostras de Variáveis Aleatórias 18

2.3 Geradores de Amostras de Variáveis Aleatórias Baseados em Congruência linear 21

2.4 Parâmetros do Gerador Baseado em Congruência linear 22

2.5 Exemplos de LCRNG 26

2.6 Interpretação Geométrica do Gerador LCRNG 29

2.7 Outros Geradores de Amostras de Variáveis Aleatórias 30

2.8 Geração de Amostras de Variáveis Aleatórias com Distribuição de Probabilidade diferente da Uniforme: Método da Transformada Inversa 31

2.9 Geração de Amostras de Variáveis Aleatórias Gaussianas 34

2.10 Testes de Geradores de Amostras de Variáveis Aleatórias 37

3 Método de Monte Carlo com Cadeia de Markov 41

3.1 Introdução $\quad 41$

3.2 Processos Estocásticos e Cadeias de Markov 42

3.3 O Algoritmo de Metropolis-Hastings Aplicado a Variáveis Aleatórias 51

3.4 O Algoritmo de Metropolis-Hastings Aplicado a Vetores Aleatórios 59

4 Vibrações Estocásticas em Sistemas com Um Grau Liberdade $\quad 65$

4.1 Introdução 65

4.2 Processos Estocásticos no Domínio da Frequência 66

4.3 Resposta ao Impulso Unitário no Domínio da Frequência 77

4.4 Resposta do Sistema para um Forçamento caracterizado como Processo Estocástico Estacionário $\quad 80$

4.5 Resposta do Sistema para um Forçamento caracterizado como um Ruído Branco $\quad 86$

5 Vibrações Estocásticas em Sistemas com Mais de Um Grau Liberdade 88

5.1 Introdução 88

5.2 Vibração Determinística de Sistemas com Mais de Um Grau de Liberdade 88

5.3 Método da Resposta ao Impulso 93

5.4 Método dos Modos Normais 99

6 Vibrações Estocásticas em Sistemas Contínuos 103

$\begin{array}{lll}6.1 & \text { Introdução } & 103\end{array}$

6.2 Modelagem de Barras e Vigas 103

6.3 O Método dos Elementos Finitos 111

6.4 Cálculo dos Modos e Frequências Naturais através dos Elementos Finitos 124 
$\begin{array}{lll}6.5 & \text { Modelo Reduzido } & 126\end{array}$

$\begin{array}{ll}\text { 6.6 Exemplo: Riser de Perfuração } & 127\end{array}$

7 Conclusão e Trabalhos Futuros $\quad \mathbf{1 3 5}$

$\begin{array}{ll}\text { Referências Bibliográficas } & 137\end{array}$

$\begin{array}{ll}\text { A Lista dos Programas MATLAB desenvolvidos } & \mathbf{1 4 0}\end{array}$

$\begin{array}{lll}\text { B Programa MATLAB de Elementos Finitos } & \mathbf{1 4 5}\end{array}$ 


\section{Lista de figuras}

2.1 Método de Monte Carlo. 17

2.2 Variável aleatória (mapa de $\Omega$ para $\mathbb{R}$ ). 18

2.3 Relação de volume entre cubo e esfera. 26

2.4 Cálculo de uma aproximação para $\pi$ através do gerador RND da IBM utilizando 10000 amostras.

2.5 Cálculo de uma aproximação para $\pi$ através do gerador antigo do MATLAB utilizando 10000 amostras.

2.6 Histograma - gerador da IBM. 29

2.7 Histograma - gerador antigo do MATLAB. 29

2.8 Interpretação Geométrica do Gerador LCRNG. 30

2.9 (a) Histograma de 10000 amostras de $X$. (b) Gráfico da função densidade de probabilidade $f(2-25)$.

2.10 (a) Gráficos de frequência de 10000 amostras de $Y_{1}$. (b) Gráficos de frequência de 10000 amostras de $Y_{2}$. 36

2.11 Limites do teste do qui-quadrado.

2.12 Função de probabilidade acumulada $P$ (de uma distribuição uniforme) e função distribuição acumulada aproximada para um experimento com 12 realizações.

2.13 Limites do teste de Kolmogorov-Smirnov

3.1 Grafo de Transição de uma cadeia de Markov com quatro estados $\left(s_{1}, s_{2}, s_{3}\right.$ e $\left.s_{4}\right)$.

3.2 Grafo de Transição de uma cadeia de Markov com duas distribuições estacionárias.

3.3 Grafo de Transição de uma cadeia de Markov com dois estados ( $s_{1}$ e $s_{2}$ ).

3.4 (a) Histograma das amostras obtidas por MCMC ( $10^{4}$ amostras).

(b) Função densidade de probabilidade gamma $\left(\sigma^{2}=\alpha \beta^{2}\right)$.

3.5 Amostras geradas de $X$ obtidas utilizando-se (a) $h$ uniforme e (b) $h$ qui-quadrado.

3.6 Função densidade de probabilidade $p$ (com $\underline{x}=3.0, \delta_{X}=0.25 \mathrm{e}$ $\left.\sigma_{X}=0.75\right)$ e as duas distribuições propostas: $h_{1}(\operatorname{com} \lambda=0.08)$ e $h_{2}(\operatorname{com} \alpha=3.0$ e $\beta=3.5)$.

3.7 Gráfico de frequência de (a) $5 \times 10^{3}$ e (b) $5 \times 10^{4}$ amostras de $X . \quad 59$

3.8 Primeiras 500 amostras da sequência $x^{(i)}$ obtidas pela distribuição proposta (a) $h_{1}$ e (b) $h_{2}$.

3.9 Vetor aleatório (mapa de $\Omega$ para $\mathbb{R}^{3}$ ).

3.10 Densidade de probabilidade Gaussiana.

3.11 Histograma para uma distribuição de probabilidade de um vetor aleatório de duas dimensões (a) $10^{4}$ e (b) $10^{6}$ amostras.

4.1 Sistema massa-mola-amortecedor submetido a uma força estocástica $F(t)$.

4.2 Representações da função densidade espectral. 
4.3 Representação (a) do espectro de um ruído branco $S_{X}(\omega)=S_{0}$ e (b) de uma realização do processo estocástico $X(t)$ ao longo do tempo.

4.4 Representação do espectro de um ruído branco $S_{X}(\omega)=S_{0}$ submetido à um filtro passa baixa com frequência de corte $\omega_{c}$.

4.5 Realização ao longo do tempo de um ruído branco simulado a partir da função densidade de probabilidade: (a) uniforme (b) normal.

4.6 Estimativa da função densidade espectral de ruído branco simulado a partir da função densidade de probabilidade: (a) uniforme (b) normal.

4.7 Representação da função impulso.

4.8 Média $\mu_{X}$ para vários valores de amortecimento do sistema $\zeta . \quad 82$

4.9 para vários valores de amortecimento $\zeta$. 84

4.10 Densidade espectral do forçamento (ruído branco).

4.11 Densidade espectral da resposta em deslocamento (força modelada como ruído branco).

5.1 Sistema com $n$ graus de liberdade.

5.2 Sistema massa-mola-amortecedor com 2 graus de liberdade.

5.3 Densidade Espectral de $S_{X_{1} X_{1}}$ e $S_{X_{2} X_{2}}$ - Método da Resposta ao Impulso

5.4 Densidade Espectral de $S_{X_{1} X_{1}}$ e $S_{X_{2} X_{2}}$ - Método dos Modos Normais 102

6.1 Exemplo de Barra fixa-livre

6.2 Seção de viga : forças cortantes e momentos.

6.3 Representação gráfica das funções $\cos$, cosh e $-1 / \cosh$

6.4 Exemplo domínio dividido em elementos lineares: funções de interpolação para os nós 4 e 5

6.5 Funções base lineares no sistema de coordenadas local.

6.6 Funções base quadráticas no sistema de coordenadas local. 117

6.7 Elemento hermitiano com dois nós. 117

6.8 Funções base hermitianas: elemento com dois nós. 119

6.9 Elemento hermitiano com três nós. 120

6.10 Funções base hermitianas: elemento com três nós. 122

6.11 Quatro primeiros modos da viga engastada-livre. 125

6.12 Aproximação dos quatro primeiros modos da viga engastada-livre (FEM) obtida com 50 elementos de Hermite de 2 nós.

6.13 Riser de perfuração de uma plataforma de petróleo.

6.14 Carregamentos aplicados no riser de perfuração.

6.15 Riser: deslocamento ao longo do tempo para $x=L$.

6.16 Riser: deslocamento ao longo do tempo para $x=L / 2$. 132

6.17 Riser: rotação ao longo do tempo para $x=L$.

6.18 Riser: rotação ao longo do tempo para $x=L / 2$. 133

6.19 Deslocamento da extremidade do riser considerando-se apenas um modo de vibração.

6.20 Deslocamento da extremidade do riser considerando-se dois modos de vibração.

B.1 Diagrama do Programa Desenvolvido. 


\section{Lista de tabelas}

2.1 Cálculo do resto de uma divisão em representação binária para divisor sendo uma potência de 2 . 22

2.2 Potências de 2 em representação decimal binária. 23

2.3 Método da Transformada Inversa aplicado a algumas distribuições. 33

2.4 Estatísticas das amostras de $X$ obtidas pelo método da Transformada Inversa.

2.5 Estatísticas das amostras de $X_{1}$ e $X_{2}$ obtidas pelo Método de BoxMuller.

3.1 Estimativas da variância da função densidade de probabilidade gamma $(\alpha=2$ e $\beta=1)$

3.2 estimativas de média e variância de $X$ calculadas a partir das amostras geradas com MCMC e com o comando gamrnd do MATLAB.

3.3 Estimativas de $m_{X}$ e $C$.

6.1 Condições de contorno e configurações de barras. 105

6.2 Condições de contorno e configurações de vigas. 109

6.3 Quadratura Gaussiana: valores de $\xi$ e $w$ em função do número de pontos utilizados para a integração.

6.4 Valores de parâmetros utilizados na simulação MATLAB: programa de elementos finitos.

6.5 Frequências Naturais do riser de perfuração associadas aos 4 primeiros modos de vibração.

$\begin{array}{lll}\text { A.1 Programas MATLAB do segundo capítulo. } & 140\end{array}$

A.2 Programas MATLAB do terceiro capítulo. 141

A.3 Programas MATLAB do quarto capítulo. 142

A.4 Programas MATLAB do quinto capítulo. 143

A.5 Programas MATLAB do sexto capítulo. 144

B.1 Correspondência entre descrição global e local para elementos hermitianos com dois nós.

B.2 Correspondência entre descrição global e local para elementos hermitianos com três nós.

B.3 Correspondência entre numeração dos graus de liberdade e a numeração global dos nós (elementos hermitianos com dois nós).

B.4 Correspondência entre numeração dos graus de liberdade e a numeração global dos nós (elementos hermitianos com três nós). 
A ciência é única ferramenta para enfrentarmos o desconhecido.

François Marie Arouet, Voltaire. 